\title{
THE MOBILITY OF THE POSITIVE AND NEGATIVE ION AT DIFFERENT TEMPERATURES AND AT CONSTANT GAS DENSITY.
}

By Henry A. Erikson.

$\mathrm{T}^{\mathrm{H}}$ HE object in pursuing the present investigation was to obtain evidence as to what change takes place in the size of the positive and negative ion in air when the temperature is altered, the density of the air being kept constant.

Evidence that a change takes place at temperatures less than $\mathrm{o}^{\circ} \mathrm{C}$. is offered by the results obtained by Phillips ${ }^{1}$ and Kovarik ${ }^{2}$ who independently determined the mobility at different temperatures. These results, however, were obtained at constant gas pressure and, therefore, do not necessarily indicate. what happens when the density is kept constant, since the conditions to which the ions are subject in the two cases are not the same.

The method used was Rutherford's alternating field method as modified by Franck and Pohl. This method, from the standpoint of convenience, is well adapted for an investigation of this kind.

Fig. $I$ is a diagram of the apparatus and experimental arrangement. $A$ is a cylindrical vessel that has a radius of $6.2 \mathrm{~cm}$. and a depth of $3.2 \mathrm{~cm}$. $B$ is a circular plate that has a radius of $5 \mathrm{~cm}$. This plate is supported by the insulated stem $C$, and through it is connected to a quadrant electrometer. In order to reduce heat conduction a portion of the stem $C$ is of glass. One centimeter above $B$ is another plate $D$, of same diameter as $B$. The opening in $D$ has a radius of $3.8 \mathrm{~cm}$. and is covered by a brass wire gauze having nine meshes to the centimeter. The ring $D$ is supported by means of three rods of quartz. A small polonium plate is attached face up at the center of the gauze. Another plate $E$ is placed $4 \mathrm{~mm}$. above $D$, and is supported and insulated from $D$ by means of the three quartz rods.

The rays from the polonium ionizes the air between the plates $D$ and $E$, which are kept at a difference of potential of about 0.8 volt by means of the battery $F$. The positive or negative ions as the case may be are

${ }^{1}$ Phillips, Proc. Roy. Soc. LXXVIII., I906, p. I67.

${ }^{2}$ Kovarik, Phys. Rev., XXX., April, I9ıо. 
driven towards the gauze through which some of them pass and thus become subject to the alternating field that is produced when the tuning fork alternately connects $D$ to the positive and negative poles of the battery $G$. The wires connected to plates $D$ and $E$ pass through two quartz tubes placed one inside the other. For determining the temperature, a constantine wire is connected to the copper wire attached to $E$. These two wires pass without touching through the outer quartz tube.

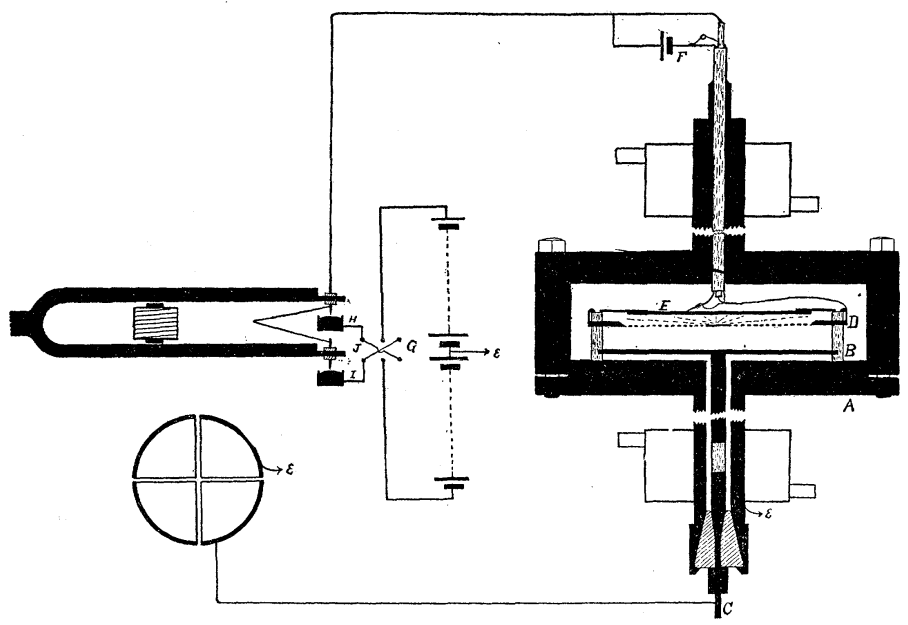

Fig. 1.

These tubes are held in place and made air tight by means of a hard wax. The ends of the upper and lower stems of the apparatus are kept at a safe temperature by means of water jackets placed as shown in figure.

This tuning fork method for shifting the polarity was found to be very satisfactory. $H$ and $I$ are two iron mercury cups of $8 \mathrm{~mm}$. diameter. Contacts with the mercury in these cups are made through steel needles attached to the prongs of the fork. It is possible to adjust so that the mercury makes no perceptible vibration thus giving regularity of contact. Since it is difficult to secure the same interval of contact for each of the points a reversing key $J$ was introduced and the average of an equal number of readings for each point was obtained.

By suitably adjusting the frequency of the fork and the potential of $G$ some of the ions will reach the plate $B$ before the field is reversed. The total charge then given to $B$ in a given time may be measured by means of the electrometer.

This arrangement gives rise to the following relation: 


$$
q=i t=i\left(\frac{t^{\prime}}{2}-\frac{d^{2} n t^{\prime}}{k v}\right)
$$

where

$i=$ the current that flows through the gauze,

$t=$ the time to charge the plate $B$ to a given potential when the field between $B$ and $D$ is constant,

$t^{\prime}=$ the time to charge the plate $B$ to the same potential when the field is alternating,

$d=$ the distance between the plates,

$n=$ the frequency of the fork,

$v=$ the difference of potential between $B$ and $D$,

$k=$ the mobility.

In this equation $d^{2} n / k v$ is the time consumed per second by the ions in passing from the gauze to the plate $B$.

From the above relation we see that:

$$
\frac{2 t}{t^{\prime}}=\mathrm{I}-\frac{2 d^{2} n}{k v}
$$

or

$$
\frac{d}{d n}\left(\frac{2 t}{t^{\prime}}\right)=\frac{2 d^{2}}{k v}, \text { a constant. }
$$

This shows that the current in the case of the alternating field which is

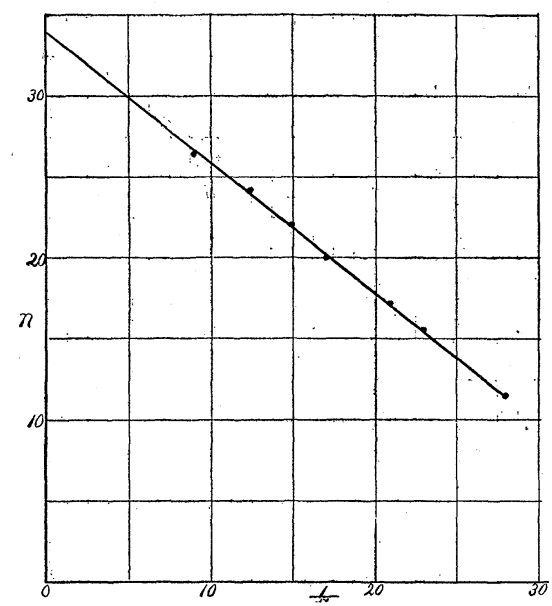

Fig. 2.

proportional to $\mathrm{I} / t^{\prime}$ is a linear function of $n$ the frequency of alternation. That this holds is borne out by experiment the results of which are shown graphically in Fig. 2. By prolonging the line until it cuts the $y$ axis we obtain the value of $n$, the frequency, for which $t^{\prime}$ becomes infinite. In terms of this frequency 


$$
k=\frac{2 d^{2} n}{V} .
$$

The above method necessitates the changing of the frequency of the fork. As this, in the case of the fork used, was not convenient, the mobility was calculated by means of the equation

$$
k=\frac{2 n d^{2} t^{\prime}}{V\left(t^{\prime}-2 t\right)},
$$

which is obtained by solving for $k$ in equation (I). Since $d, n$ and $v$ are kept the same they may be determined once for all, and therefore only $t$ and $t^{\prime}$ remain to be determined at the different temperatures. It was found necessary to determine $t$ at each temperature because it does not remain constant as the temperature is changed. This change is undoubtedly due to a greater diffusion of the ions to the gauze as the temperature is raised. The value of $t$ is greater for the negative ions than for the positive. This is what should be expected in view of the greater diffusion of the negative ions. Another effect is noticed in the case of $t$. As the voltage applied from the battery $F$ is increased from zero, the current at first increases and then diminishes. The reason for this decrease of the current through the gauze is undoubtedly due to the greater number of ions drawn to the gauze at the higher voltage values. By measuring $t$ at each temperature the method becomes independent of these changes. There are, however, several possible sources of error that must be considered. When the temperature is altered convection currents arise. Care was taken to have the vessel well surrounded by the heating or cooling medium. The metal wall of the vessel is $1.3 \mathrm{~cm}$. thick, which also aids in securing uniformity of temperature. Furthermore since the field employed, $\mathrm{I} 20$ volts to the $\mathrm{cm}$., was such as give the ions a velocity of over $100 \mathrm{~cm}$. per second the convection currents present in this case can have but a small effect. Tests made under conditions most favorable for these currents seemed to show no effect. The effect of diffusion to the sides owing to the nearness of the plates is negligible.

An uncertainty enters in connection with the conditions at the gauze at the different temperatures. During the application of the reverse field the ions are driven back giving rise to a greater ionic density near the gauze which when the direct field comes on may result in a greater current through the gauze than the steady current measured by $t$. Also since the diffusion is different at the different temperatures the ions may not have the same starting point under all conditions. Furthermore, there is an uncertainty in connection with the potential gradient at the 
gauze and plate $B$. How important these considerations are is difficult to say. The method seems to give good values as compared with other methods. In the table below are given the results, in the order obtained, for the mobility of the positive and negative ions over a temperature range from $-2 \mathbf{1}^{\circ} \mathrm{C}$. to $63^{\circ} \mathrm{C}$. The air involved in this set was dryed by passing through a tower of $\mathrm{CaCl}_{2}$ and then through a specially designed drying tube immersed in liquid air. Before leaving this tube the air passed through glass wool. The chamber before filling was left evacuated for twelve hours and was also evacuated several times during filling with the dry air. The vessel, however, was not heated during the process of filling.

The pressure of the air was $73.5 \mathrm{~cm}$. at $24.5^{\circ} \mathrm{C}$.

TABLE I.

$+$

\begin{tabular}{|c|c|c|c|c|c|c|}
\hline-184 & $-2 I$ & 0 & 24 & 33 & 45 & 63 \\
\hline & $\begin{array}{l}1.321 \\
1.321 \\
1.322\end{array}$ & $\begin{array}{l}1.327 \\
1.359 \\
1.368 \\
1.392\end{array}$ & $\begin{array}{l}1.375 \\
1.366 \\
1.334 \\
1.327 \\
\\
1.366 \\
1.379 \\
1.404\end{array}$ & $\begin{array}{l}1.340 \\
1.305 \\
1.333\end{array}$ & $\begin{array}{l}1.318 \\
1.307 \\
1.208\end{array}$ & $\begin{array}{l}1.193 \\
1.213 \\
1.216\end{array}$ \\
\hline 1.20 & 1.321 & 1.361 & 1.364 & 1.326 & 1.278 & 1.207 \\
\hline
\end{tabular}

\begin{tabular}{c|c|c|c|c|c|c}
\hline$-\mathrm{r} 84$ & $-2 \mathrm{r}$ & $\mathrm{0}$ & 24 & 33 & 45 & 63 \\
\hline & & & 1.833 & 1.801 & 1.807 & 1.721 \\
& & & 1.794 & 1.768 & 1.827 & 1.790 \\
& & & 1.750 & 1.763 & 1.795 & 1.677 \\
& & 1.602 & 1.707 & & & \\
& & 1.503 & & & & \\
& & 1.495 & & & & \\
& 1.638 & 1.490 & 1.666 & & & \\
\hline 1.24 & 1.543 & & 1.576 & & & \\
\hline
\end{tabular}

In Fig. 3 the average values are shown graphically by curves $A$ and $B$. It is seen that the positive mobility is a maximum between the room 
temperatures and zero degrees. The different values are quite regular and may be repeated with fair agreement.

The negative values, however, are quite erratic. It is seen that the ratio $k_{-} / k_{+}$is normal at room temperature just after filling with the dry air. As the temperature was raised the value of the mobility diminished. When the vessel had cooled to room temperature it was found that the mobility was less than the value obtained at room temperature before heating and the same effect repeated itself after each successive heating. Apparently vapor is liberated from the apparatus during

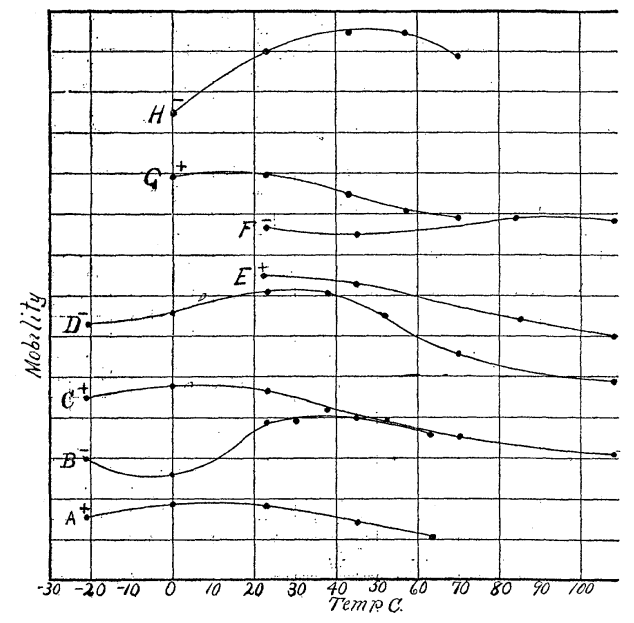

Fig. 3.

heating that reduces the mobility. Upon cooling to zero there is a large decrease in the mobility. Upon further cooling the mobility is greater than at zero indicating a dryer state due to deposit of moisture on walls of vessel.

After the above readings were obtained the vessel was evacuated and kept at a temperature well over Ioo C. for an hour or more. It was then evacuated filled several times while hot with air dried by passing through $\mathrm{CaCl}_{2}$ and the tube immersed in liquid air. In Table II. are given the results obtained with this sample of air. The results are arranged in the order obtained beginning in each case with the value at $24^{\circ} \mathrm{C}$.

These results are shown graphically in Fig. 3 by means of the curves $C$ and $D$. In this case the variation of the mobility of the positive ion is quite similar to that of the preceding case except that the diminution is somewhat greater at the higher temperatures. In the case of the negative ion there is a less sudden decrease from room temperature to 
TABLE II.

\begin{tabular}{|c|c|c|c|c|c|}
\hline$-2 \mathrm{I}$ & ○ & 24 & $3^{8}$ & $5^{2}$ & 70 \\
\hline 1.30 & 1.365 & $\begin{array}{r}1.361 \\
1.330 \\
1.322 \\
1.331 \\
1.327\end{array}$ & $\begin{array}{l}1.206 \\
1.212 \\
1.209\end{array}$ & $\begin{array}{l}1.142 \\
1.206 \\
1.178\end{array}$ & $\begin{array}{l}1.096 \\
\\
1.107 \\
1.101\end{array}$ \\
\hline
\end{tabular}

\begin{tabular}{c|c|c|c|c|c}
\hline-21 & 0 & 24 & $38^{\circ}$ & 52 & 70 \\
\hline & & 1.882 & 1.84 & 1.687 & 1.534 \\
& 1.755 & 1.825 & & & \\
& & 1.812 & 1.78 & 1.716 & \\
1.663 & & 1.685 & & & 1.487 \\
& & 1.740 & 1.81 & 1.701 & 1.510 \\
\hline
\end{tabular}

zero but a greater decrease at the higher temperatures. The vessel was then evacuated and filled with air that had been allowed to bubble through water before entering the chamber. The results obtained are given in the Table III.

\begin{tabular}{l|c|c|c|c|c|c|c}
\multicolumn{2}{c}{+ TABLE III. } \\
\hline 24 & 45 & 84 & ro & 24 & 45 & 84 & ro \\
\hline 1.321 & & & & 1.560 & & & \\
1.326 & & 1.12 & & 1.592 & & 1.625 & \\
1.330 & 1.295 & & 1.042 & 1.547 & 1.543 & & 1.600 \\
\hline
\end{tabular}

These results are shown graphically by means of curves $E$ and $F$ in Fig. 3 .

It is seen that the variation of the positive mobility is in this case about the same as in the case of the drier air used in the two preceding cases. The negative shows a maximum at a higher temperature but on the whole does not vary greatly over the temperature range employed. On account of deposit of moisture on the insulation no values for zero degrees or lower were obtained.

The vessel was now evacuated and heated to about $100^{\circ} \mathrm{C}$. and kept at this temperature for a period of about five hours. During this period the pump was operating constantly. A very small stream of air from a tower of $\mathrm{CaCl}_{2}$ was allowed to pass through the vessel during this time. Finally the vessel was evacuated while hot, filled several times with air passed slowly through the tube immersed in liquid air. 
In the Table IV. are given the results obtained in this case.

TABLE IV.

\begin{tabular}{c|c|c|c|c|c|c|c}
\multicolumn{1}{c}{ - } \\
\hline${ }_{24}$ & 43 & 57 & 70 & 24 & 43 & 57 & 70 \\
\hline 1.398 & 1.287 & 1.206 & & 2.032 & 2.094 & 2.080 & \\
1.366 & & & 1.167 & 1.846 & & & 1.959 \\
\hline
\end{tabular}

These results are shown graphically by means of curves $G$ and $H$ in Fig. 3. It is seen that the variation of the positive mobility is about the same as obtained in the preceding cases. The negative shows a marked variation and has a maximum at a higher temperature than before.

\section{Discussion of Results.}

In seeking an explanation for the variation of the mobility of the positive ion the question of clustering immediately presents itself. Since, however, the mobility of the positive ion is quite independent of the presence of moisture and also since the variation with temperature is also quite independent of the condition of the air, an explanation based on clustering seems difficult. It is possible that something is driven from the wall of the vessel during heating for which the positive ion has a special attraction. It thereby becomes loaded and moves more slowly. It hardly seems probable, however, that it would unload again as the temperature is lowered; moreover this would not explain the decrease that takes place below the temperature for which the mobility is a maximum. Definite conclusion on this point will have to be postponed until a more rigid investigation has been made. The variation of the mobility of the negative ion seems to show the effect of clustering due to moisture combined with a variation similar to that observed in the case of the positive ion. Not sufficient data has been obtained, however, upon which to base any conclusion.

In connection with the observed variations an indication based on

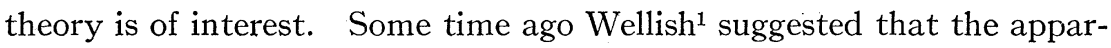
ently large volume of the ions is due to the effect of the charge upon the mean free path, and showed that the polarization of the adjacent molecules due to the ionic charge results in an apparent increase in the radius of the sphere of action. A little later Sutherland ${ }^{2}$ by taking into account cohesion and electrical attraction evolved the following expression for

1 Wellish, Phil. Trans., A, Vol. 209, p. 249, January, I909.

${ }^{2}$ Sutherland, Phil. Mag., 6-r8, September, 1909. 
the mobility of an ion moving in a gas under the action of an electric field.

$$
k=\frac{e \frac{d E}{d x}}{\frac{4}{3} N_{3}\left(a_{1}+a_{3}\right)^{2}\left(\frac{2 \pi}{3} \frac{m_{1} m_{3}}{m_{1}+m_{3}} m_{3} v_{3}\right)^{\frac{1}{2}}\left\{\mathrm{I}+\frac{\varphi\left(a_{1}+a_{3}\right)+\theta\left(a_{1}+a_{3}\right)}{\frac{m_{3} v_{3}^{2}}{2}}\right\}},
$$

where

$N_{3}=$ number of molecules per $\mathrm{cm} .{ }^{3}$

$a_{1}+a_{3}=$ the distance an ion and molecule must approach for collision, $m_{1}$ and $m_{3}=$ mass of ion and molecule respectively,

$\varphi\left(a_{1}+a_{3}\right)$ and $\theta\left(a_{1}+a_{3}\right)$ are functions depending respectively upon cohesion and electric action.

By considering $N_{3}$ constant and placing

$$
\frac{m v_{3}^{2}}{2}=k T,
$$

where $T$ is the absolute temperature, there results for the mobility

$$
k=\frac{A \sqrt{T}}{T+C} .
$$

By differentiating with respect to $T$ and equating to zero we see that $k$ is a maximum when $T=C$. The variation obtained, however, is greater than expressed by this equation.

\section{SUMMARY.}

The mobility of the positive and negative ions in air at atmospheric density has been measured at different temperatures by means of an alternating field method.

It was found that under the above stated conditions the mobility of the positive ion is a maximum at about $o^{\circ} \mathrm{C}$. The mobility of the negative ion also shows a maximum but is more erratic in its behavior. No definite conclusion has been reached but it seems that the variation of the positive mobility is not due to clustering. The variation of the negative mobility, however, seems to be due to clustering combined with an effect similar to that causing the variation of the positive mobility.

In connection with this investigation I wish to express my thanks to Professor John Zeleny and Professor A. F. Kovarik.

Physical Laboratory, University OF Minnesota,

Mạy, I9I5. 